\title{
Evaluation of Natural and Chemical Phosphorus Fertilizers on Plant Growth, and Yield of Some Green Beans Cultivars
}

\author{
M.S.A. Emam, Noha G. Abd El-Rahman and M. A. A. Abdrabbo \\ Central Laboratory for Agricultural Climate, Agricultural Research Centre, Giza, Egypt
}

Correspondence author: dr.nohagaber@yahoo.com

\begin{abstract}
A field experiment was conducted during the two successive seasons of 2015/2016 and 2016/2017 at Central Laboratory for Agricultural Climate (CLAC), Agricultural Research Centre, Dokki, Giza governorate, Egypt to study the effect of two green bean cultivars (Paulista and Samantha) and three rates of rock phosphate (fine and granules) as a natural resource of $\mathrm{P}$ mixed with sand substrate in comparison with calcium superphosphate with recommended level on growth and yield of green bean (Phaseolus vulgaris L.) under plastic house conditions. Regarding the green bean cultivars, Paulista gave higher plant height, number of leaves, canopy and root fresh and dry weight than Samantha cultivar. Paulista cultivar produced the highest values of total pod yield per plant during the two successive seasons. Increasing phosphorus level (fine or granule rock phosphate) up to $150 \%$ enhanced pod yield with both used cultivars. The highest vegetative growth and yield were obtained from 150\% fine rock phosphate combined with Paulista cultivar. Tissue nutrient analysis show that increase phosphorus level by rock phosphate source led to increase nutrient percentage for NPK in green beans leaves in comparison with recommended phosphorus dose applied as calcium superphosphate. The economic consideration among phosphorus and different $\mathrm{P}$ sources revealed that fine rock phosphate with Paulista cultivar gave the highest net income per greenhouse.
\end{abstract}

Keywords: Natural fertilizer, phosphorus use efficiency, plant analysis, vegetables and economical consideration.

\section{Introduction}

The green bean (Phaseolus vulgaris L.) is widely grown around the world; it is one of the most important nutritious crops for human consumption. In general, green bean in legume family has the first rank in the world according to FAO Stat (2013). The nutritional benefits and contribution of beans to human health is recognized targeting human ailments like cancer, diabetes and heart diseases (Haugen and Bennink, 2003). Phosphorus (P) is the major essential macronutrient in soils after nitrogen $(\mathrm{N})$ which plays an important role in plant development and growth. Phosphorus involved in many reactions in plants such as energy transfer, photosynthesis, respiration, as well as transfer of genetic characteristics in plants (Johnston and Steen, 2000).Poor soil fertility, particularly phosphorus, consider one of the main factors responsible for low crop yields in arid regions. Total $\mathrm{P}$ in soils is very high, but is very low available (Liu et al., 1994). Only around $10 \%$ of applied $P$ is immediately available for plants, the rest converted into insoluble compounds or adsorbed to soil particles (Mongi, 1974). Phosphorus fertilization improves quality of vegetable fruits, and grain crops because increases their resistance to diseases as well as drought and adverse environmental conditions. Phosphorus is a major component of compounds whose functions which relate to plant growth and productivity (Raboy, 2003). Phosphorus is one of the most sufficiently determinants of plant growth (Wang et al., 1998). Growth and development of plants depends largely on the development of root system.
Phosphorus intensify root growth, which improves the water and other nutrients absorption and translocation to the growing parts of the plants, resulting in an increased photosynthetic process and then better production. (Robinson et al., 1981) reported the effect of phosphorus in stimulating root and plant growth, initiation of nodule formation as well as influencing the general efficiency of the rhizobium bacteria.

The availability of plant nutrients in soil depends on soil chemical and physical properties. P deficiency reduces plant growth and crop productions especially in arid and semi-arid areas with calcareous soils (Bargaz et al., 2016). (Hinsinger, 2001) reported that the two forms of phosphorus in soil are organic and mineral. Organic phosphorus is the more available form of phosphorus in the soil. While inorganic phosphorus is stable. Therefore, mineral phosphorus is readily absorbed and used by crop if it is not fixed. (Nikolay Vassilev et al., 1996) reported that organic phosphorus form is mineralized and immobilized by microbes' activities. Mineralization is the conversion of organic phosphorus to inorganic phosphorus, while, the immobilization of phosphorus involves the formation of organic phosphorus from inorganic phosphorus.

Rock phosphate (RP) is one of the indigenous phosphate sources in many countries. The phosphorus content in the rock is ranged from 25 to $31 \% \mathrm{P}_{2} \mathrm{O}_{5}$ with low solubility (Adetunji, 2005). Application of rock phosphate has been proved to be beneficial to plant growth and productivity (Akande $\boldsymbol{e t}$. al., 2008). The methods of enhancing the quality of phosphate rock and its agronomic efficiency include composting RP 
with organic manures, partially acidulating with mineral acids and compacting with super phosphate (Adediran and Sobulo, 1997).

The objective of this study was to investigate the growth and productivity of green beans under different applications of different sources of $\mathrm{P}$ in sandy culture under plastic house conditions.

\section{Materials and methods}

This experiment was conducted at Central Laboratory for Agricultural Climate (CLAC), Agriculture Research Centre, Dokki, Giza governorate, Egypt under plastic house conditions (9m width $\times 60 \mathrm{~m}$ length $\times 3.25 \mathrm{~m}$ height) during the two successive seasons of 2015/2016 and 2016/2017.

\section{Plant material:}

Seeds were obtained from Sueze Canal Company for agricultural and commercial development. Two cultivars (Paulista and Samantha) were sown on first of November in the both cultivated seasons. Two seeds were planted directly in each pot.

\section{System materials:}

Plastic pots $8 \mathrm{~L}$ ( $25 \mathrm{~cm}$ diameter $\times 30 \mathrm{~cm}$ height) were used, the pots were filled by sand in open system of sand culture. Sand was primarily washed with diluted nitric acid to get rid from the undesirable salts, then with running tap water to wash nitric acid compounds from the sand.

After sand was getting dry, it mixed with Rock Phosphate in different rates regarding to the treatments under the study and the pots were filled in specific volume $8 \mathrm{~L}$. Black polyethylene sheets were mulched the soil of unheated plastic house before

The pots were arranged in rows. The final plant spacing was $30 \mathrm{~cm}$ in the row, $60 \mathrm{~cm}$ between the rows and between the double rows was $70 \mathrm{~cm}$. The chemical fertilizers were injected with drip irrigation system. Sub-miserable pump (110 watt) at water tank $120 \mathrm{~L}$ for each experimental plot was used to pump the fertigation via emitters' $4 \mathrm{l} / \mathrm{hr}$ capacity. The fertigation was programmed to work 4 times / day and the duration of irrigation time depended upon the season. All the other agricultural practices of green bean cultivation were carried out according to Ministry of Agriculture and Land Reclamation (2013).

\section{The studied treatments:}

The experiment was designed to study the effect of three factors on snap bean yield as follows:

1. Three rates of rock phosphate as a natural resource of phosphorus mixed with sand substrate calculated on the standard recommended of green bean program fertilization and the rock phosphate content of $\mathrm{P}$ as follows:

a. $100 \%$ of recommended $=10.0 \mathrm{~g}$ fine or granule rock phosphate / plant $(\mathrm{P}-100 \%)$

b. $125 \%$ of recommended $=12.5 \mathrm{~g}$ fine or granule rock phosphate / plant $(\mathrm{P}-125 \%)$

c. $150 \%$ of recommended $=15.0 \mathrm{~g}$ fine or granule rock phosphate / plant (P-150\%)

d. The control (using super phosphate $\left(15.5 \% \mathrm{P}_{2} \mathrm{O}_{5}\right)$ during plant growth period according to the short snap bean program fertilization (40 units of $\mathrm{P}_{2} \mathrm{O}_{5}$ during season)

Table 1. Chemical constituents of rock phosphate.

\begin{tabular}{cccccccc}
\hline & $\mathrm{SiO}_{2}$ & $\mathbf{T i O}_{2}$ & $\mathbf{A l}_{2} \mathbf{O}_{3}$ & $\mathbf{F e}_{2} \mathbf{O}_{3}$ & $\mathbf{M n O}$ & $\mathbf{M g O}$ & $\mathbf{C a O}$ \\
\cline { 2 - 8 } Component \% & 19.15 & 0.06 & 1.13 & 0.89 & 0.02 & 0.22 & 41.25 \\
\cline { 2 - 8 } & $\mathbf{N a 2} \mathbf{O}$ & $\mathbf{K}_{2} \mathbf{O}$ & $\mathbf{P}_{2} \mathbf{O}_{5}$ & $\mathbf{C L}$ & $\mathbf{S O}_{3}$ & $\mathbf{F}$ & L.O.I \\
\cline { 2 - 9 } & 0.57 & 0.07 & 20.95 & 0.39 & 1.21 & 1.94 & 11.50 \\
\hline
\end{tabular}

\section{Measurements}

Samples of plants of each experimental treatment were taken to determine growth parameters at 60 days from the sowing as follows:

1. Number of leaves.

2. Plant height.

3. Stem diameter.

4. Vegetative fresh weight.

5. Vegetative dry weight.

6. Root fresh weight.

7. Root dry weight.

Yield:

Total yield per plant was recorded as g/plant.

Pods quality:

1. Pod weight.

2. Pod length.

3. Pod diameter.

4. Fibers content.

\section{Chemical properties of pods were determined}

1. Fibers were determined in dry samples according to A.O.A.C., (1990).

\section{Leaf mineral content:}

For mineral analysis, wet digestion was performed according to FAO SOILS BULLETIN: $0.5 \mathrm{~g}$ oven dried plant material was added in $50 \mathrm{ml}$ volumetric flask and digested with $10 \mathrm{ml} \mathrm{H}_{2} \mathrm{SO}_{4}$ conc. on a hot plate at approximately $270{ }^{\circ} \mathrm{C}$. Few drops of $\mathrm{H}_{2} \mathrm{O}_{2}$ was added repeatedly until the digest remains clear. The solution left to cool and diluted to $50 \mathrm{ml}$ with redistilled water according to the method described by Allen (1974).

1. Total nitrogen was determined by Kjeldahl method according to the procedure described by FAO (1980). 
2. Phosphorus content was determined using spectrophotometer according to Watanabe and Olsen (1965).

3. Potassium content was determined photometrically using Flame photometer as described by Chapman and Pratt (1961).

\section{Statistical analysis:}

The treatments were arranged as a factorial experiment in randomized complete blocks designed with three replications. Analysis of the data was determined by computer, using SAS program for statistical analysis and the differences among means for all traits were tested for significance at $5 \%$ level according to the procedure described by (Waller and Duncan 1969).

Economical considerations:

The economical analysis after considering the cost of phosphorus application for one greenhouse (1200 $\mathrm{m}^{2}$ ). The incomes from potato yield were used (CIMMYT, 1988) according to the formula:

Net Income $=$ value of obtained yield - cost of cultivation inputs and practices

\section{Results and discussion \\ Vegetative characters:}

The application of different rates of fine and granule rock phosphate had significant effect on plant heights of the two varieties (Paulista and Samantha) during the two studied seasons (Table 2). Paulista cultivar produced higher plant height, number of leaves per plant, canopy and roots fresh and dry weight (Table 2 and 3) values than Samantha cultivar during the both seasons. The highest plant height, number of leaves, canopy and roots fresh and dry weight were obtained by $150 \%$ (the highest $\mathrm{P}$ application) followed by $125 \%$ of recommended $\mathrm{P}$ dose for both fine and granule rock phosphate, the control (100\% P applied by calcium super phosphate) produced the lowest plant growth (plant height, number of leaves, fresh and dry weight). Stem diameter of green bean show that there were no significant differences between rock phosphate levels, while all rock phosphate treatments had significant difference compared to control treatment. There were no significant differences between Paulista and Samantha cultivars in terms of stem diameter. The same trend was obtained during second season.

Table 2. Effect of application of different rates of rock phosphate forms as well as two green bean cultivars on plant height, number of leaves and stem diameter.

\begin{tabular}{|c|c|c|c|c|c|c|}
\hline & \multicolumn{2}{|c|}{ First season 2015/2016 } & \multicolumn{3}{|c|}{ Second season $2016 / 2017$} & \multirow[b]{2}{*}{ Means } \\
\hline Treatments & Paulista & Samantha & Means & Paulista & Samantha & \\
\hline \multicolumn{7}{|c|}{ plant height (cm) } \\
\hline Granul 100\% & $44.8 \mathrm{de}$ & $45.3 \mathrm{~d}$ & 45.0 CD & $47.4 \mathrm{de}$ & $48.0 \mathrm{~d}$ & 47.7 CD \\
\hline Fine 100\% & $46.4 \mathrm{~cd}$ & $45.3 \mathrm{~d}$ & 45.9 CD & $49.2 \mathrm{~cd}$ & $48.1 \mathrm{~d}$ & 48.6 CD \\
\hline Granul 125\% & $47.7 \mathrm{c}$ & $47.7 \mathrm{c}$ & $47.7 \mathrm{~B}$ & $50.3 \mathrm{c}$ & $50.4 \mathrm{c}$ & $50.5 \mathrm{~B}$ \\
\hline Fine $125 \%$ & $47.8 \mathrm{bc}$ & $44.3 \mathrm{de}$ & $46.1 \mathrm{C}$ & $50.7 \mathrm{bc}$ & $47.0 \mathrm{de}$ & $48.9 \mathrm{C}$ \\
\hline Granul $150 \%$ & $49.4 \mathrm{bc}$ & $47.8 \mathrm{bc}$ & $48.6 \mathrm{~B}$ & $52.8 \mathrm{bc}$ & $50.7 \mathrm{bc}$ & $51.6 \mathrm{~B}$ \\
\hline Fine $150 \%$ & $52.6 \mathrm{a}$ & $49.6 \mathrm{~b}$ & $51.1 \mathrm{~A}$ & $55.8 \mathrm{a}$ & $52.6 \mathrm{~b}$ & $54.2 \mathrm{~A}$ \\
\hline Control & $43.3 \mathrm{e}$ & $46.0 \mathrm{~cd}$ & 44.7 D & $45.9 \mathrm{e}$ & $48.8 \mathrm{~cd}$ & 47.4 D \\
\hline Means & $47.4 \mathrm{~A}^{\prime}$ & $46.6 \mathrm{~B}^{\prime}$ & & $50.3 \mathrm{~A}^{\prime}$ & 49.4 B' & \\
\hline \multicolumn{7}{|c|}{ Number of leaves } \\
\hline Granul $100 \%$ & $53.3 \mathrm{bc}$ & $47.7 \mathrm{de}$ & $50.5 \mathrm{C}$ & $53.9 \mathrm{~cd}$ & $49.5 \mathrm{de}$ & $51.7 \mathrm{C}$ \\
\hline Fine $100 \%$ & $58.7 \mathrm{ab}$ & $48.3 \mathrm{de}$ & $53.5 \mathrm{BC}$ & $55.5 \mathrm{c}$ & $49.6 \mathrm{de}$ & $52.5 \mathrm{C}$ \\
\hline Granul $125 \%$ & $58.0 \mathrm{ab}$ & $48.3 \mathrm{de}$ & $53.2 \mathrm{BC}$ & $61.2 \mathrm{~b}$ & $51.2 \mathrm{~d}$ & $59.2 \mathrm{~B}$ \\
\hline Fine $125 \%$ & $62.7 \mathrm{a}$ & $51.3 \mathrm{~cd}$ & $57.0 \mathrm{AB}$ & $65.4 \mathrm{a}$ & $54.4 \mathrm{~cd}$ & 59.9 A \\
\hline Granul $150 \%$ & $61.0 \mathrm{a}$ & $51.7 \mathrm{~cd}$ & $56.3 \mathrm{AB}$ & $65.3 \mathrm{a}$ & $55.4 \mathrm{c}$ & $60.4 \mathrm{~A}$ \\
\hline Fine $150 \%$ & $60.3 \mathrm{a}$ & $54.7 \mathrm{bc}$ & $57.5 \mathrm{~A}$ & $68.0 \mathrm{a}$ & $56.0 \mathrm{c}$ & $61.8 \mathrm{~A}$ \\
\hline Control & $48.0 \mathrm{de}$ & $43.7 \mathrm{e}$ & $45.8 \mathrm{C}$ & $50.9 \mathrm{~d}$ & $46.3 \mathrm{e}$ & 48.7 D \\
\hline Means & $57.4 \mathrm{~A}^{\prime}$ & 49.4 B' & & $60.0 \mathrm{~A}^{\prime}$ & $51.8 \mathrm{~B}^{\prime}$ & \\
\hline \multicolumn{7}{|c|}{ Stem diameter $(\mathrm{cm})$} \\
\hline Granul $100 \%$ & 1.13 abe & 1.13 abe & $1.13 \mathrm{~A}$ & 1.20 abe & 1.19 abe & $1.19 \mathrm{~A}$ \\
\hline Fine $100 \%$ & $1.17 \mathrm{ab}$ & $1.13 \mathrm{abe}$ & $1.15 \mathrm{~A}$ & $1.24 \mathrm{ab}$ & 1.19 abe & $1.21 \mathrm{~A}$ \\
\hline Granul $125 \%$ & $1.17 \mathrm{ab}$ & $1.15 \mathrm{abe}$ & $1.16 \mathrm{~A}$ & $1.24 \mathrm{ab}$ & $1.24 \mathrm{abe}$ & $1.24 \mathrm{~A}$ \\
\hline Fine $125 \%$ & $1.20 \mathrm{a}$ & 1.13 abe & $1.16 \mathrm{~A}$ & $1.20 \mathrm{a}$ & 1.20 abe & $1.24 \mathrm{~A}$ \\
\hline Granul 150\% & $1.20 \mathrm{a}$ & 1.10 abe & $1.15 \mathrm{~A}$ & $1.27 \mathrm{a}$ & 1.17 abe & $1.23 \mathrm{~A}$ \\
\hline Fine $150 \%$ & $1.27 \mathrm{a}$ & $1.20 \mathrm{a}$ & $1.22 \mathrm{~A}$ & $1.28 \mathrm{a}$ & $1.27 \mathrm{a}$ & $1.31 \mathrm{~A}$ \\
\hline Control & 0.97 be & $0.90 \mathrm{e}$ & 0.95 B & 1.03 be & $0.99 \mathrm{e}$ & $1.02 \mathrm{~B}$ \\
\hline Means & $1.16 \mathrm{~A}^{\prime}$ & $1.11 \mathrm{~A}^{\prime}$ & & $1.23 \mathrm{~A}^{\prime}$ & $1.17 \mathrm{~A}^{\prime}$ & \\
\hline
\end{tabular}

It could be concluded that Samantha cultivar had significantly lower plant height, number of leaves, canopy and roots fresh and dry weight than Paulista as well as increasing application of rock phosphate up to
$150 \%$ led to increase measured vegetative characters in comparison with control. Similar observations were reported by Raghothama (1999) who concluded that sufficient $\mathrm{P}$ makes efforts to increase physiological 
process and dry matter accumulation of root and shoot. Where as inadequate supply of $\mathrm{P}$ led to slow the growth of the plant by reducing the water uptake of the root system. The role of phosphorus in enhancing root growth, which in turn improves the ability of crops for phosphate acquisition. Phosphorus application in green beans is known to have positive effects on enhancing root proliferation and thus enhancing soil water and nutrients uptake (Haugen and Bennink, 2003). Liu et al., (1994) observed that at early growth stages, plants use seed reserves for their establishment and little utilization of phosphorus to develop root system, which can scavenge the nutrients around themselves. Thus higher root development would be an important trait for adapted genotypes in exploring large soil volume hence soil moisture Hossain and Hamid (2007). Tesfaye et al., (2007) reported that there were significant increase in number of pods per green beans plants, due to increased $\mathrm{P}$ fertilization. Thus the increment of number of pods per plant due to application of phosphorus fertilizers confirms with phosphorus fertilizer promotes formation of nodes and pods in legumes.

Table 3. Effect of application of different rates of rock phosphate forms as well as two green bean cultivars on plant fresh and dry weight, root fresh and dry weight.

\begin{tabular}{|c|c|c|c|c|c|c|}
\hline & \multicolumn{2}{|c|}{ First season 2015/2016 } & \multicolumn{3}{|c|}{ Second season $2016 / 2017$} & \multirow[b]{2}{*}{ Means } \\
\hline Treatments & Paulista & Samantha & Means & Paulista & Samantha a & \\
\hline \multicolumn{7}{|c|}{ Plant fresh weight (g) } \\
\hline Granul 100\% & $326.3 \mathrm{~d}$ & $242.2 \mathrm{i}$ & $284.4 \mathrm{~F}$ & $328.2 \mathrm{~d}$ & $244.1 \mathrm{i}$ & $286.6 \mathrm{~F}$ \\
\hline Fine $100 \%$ & $333.1 \mathrm{~d}$ & $272.1 \mathrm{~g}$ & $302.5 \mathrm{E}$ & $334.2 \mathrm{~cd}$ & $273.9 \mathrm{~g}$ & $304.3 \mathrm{E}$ \\
\hline Granul 125\% & $350.4 \mathrm{c}$ & $289.1 \mathrm{f}$ & $320.3 \mathrm{D}$ & $352.5 \mathrm{c}$ & $290.3 \mathrm{f}$ & $322.4 \mathrm{D}$ \\
\hline Fine $125 \%$ & $345.1 \mathrm{~cd}$ & $310.3 \mathrm{e}$ & $328.3 \mathrm{C}$ & $347.3 \mathrm{c}$ & $312.5 \mathrm{e}$ & $330.1 \mathrm{C}$ \\
\hline Granul $150 \%$ & $365.3 \mathrm{~b}$ & $375.2 \mathrm{~b}$ & $370.4 \mathrm{~B}$ & $368.1 \mathrm{~b}$ & $378.2 \mathrm{~b}$ & 374.2 B \\
\hline Fine $150 \%$ & $390.2 \mathrm{a}$ & $382.5 \mathrm{ab}$ & $386.6 \mathrm{~A}$ & $393.5 \mathrm{a}$ & $385.3 \mathrm{~b}$ & $389.6 \mathrm{~A}$ \\
\hline Control & $259.2 \mathrm{~h}$ & $280.4 \mathrm{fg}$ & $269.2 \mathrm{G}$ & $260.4 \mathrm{~h}$ & $281.5 \mathrm{fg}$ & $271.3 \mathrm{G}$ \\
\hline Means & $338.5 \mathrm{~A}^{\prime}$ & 307.4 B' & & $340.6 \mathrm{~A}^{\prime}$ & 309.5 B' & \\
\hline \multicolumn{7}{|c|}{ Plant dry weight $(\mathrm{g})$} \\
\hline Granul $100 \%$ & $77.9 \mathrm{c}$ & $51.7 \mathrm{f}$ & $64.8 \mathrm{EF}$ & $78.3 \mathrm{c}$ & $52.1 \mathrm{~h}$ & $65.2 \mathrm{EF}$ \\
\hline Fine $100 \%$ & $76.3 \mathrm{c}$ & $57.3 \mathrm{e}$ & $66.8 \mathrm{DE}$ & $76.7 \mathrm{c}$ & $57.7 \mathrm{f}$ & 67.2 DE \\
\hline Granul 125\% & $75.1 \mathrm{c}$ & $61.7 \mathrm{de}$ & 68.4 D & $75.7 \mathrm{c}$ & $62.0 \mathrm{e}$ & 68.8 D \\
\hline Fine $125 \%$ & $75.3 \mathrm{c}$ & $68.0 \mathrm{~d}$ & $71.6 \mathrm{C}$ & $75.8 \mathrm{c}$ & $68.4 \mathrm{~d}$ & $72.1 \mathrm{C}$ \\
\hline Granul 150\% & $77.7 \mathrm{c}$ & $82.5 \mathrm{~b}$ & $80.1 \mathrm{~B}$ & $78.2 \mathrm{c}$ & $83.2 \mathrm{~b}$ & 80.7 B \\
\hline Fine $150 \%$ & $90.6 \mathrm{a}$ & $84.8 \mathrm{~b}$ & $87.7 \mathrm{~A}$ & $91.2 \mathrm{a}$ & $85.5 \mathrm{~b}$ & 88.4 A \\
\hline Control & $65.0 \mathrm{~d}$ & $59.7 \mathrm{e}$ & $62.3 \mathrm{~F}$ & $65.3 \mathrm{de}$ & 60.0 ef & $62.6 \mathrm{~F}$ \\
\hline Means & $76.8 \mathrm{~A}^{\prime}$ & $66.5 \mathrm{~B}^{\prime}$ & & $77.3 \mathrm{~A}^{\prime}$ & $66.9 \mathrm{~B}^{\prime}$ & \\
\hline \multicolumn{7}{|c|}{ Root fresh weight (g) } \\
\hline Granul 100\% & $156.3 \mathrm{~g}$ & $158.5 \mathrm{~g}$ & $157.4 \mathrm{~F}$ & $159.4 \mathrm{gh}$ & $156.4 \mathrm{~h}$ & $158.4 \mathrm{~F}$ \\
\hline Fine $100 \%$ & $172.5 \mathrm{fg}$ & $164.3 \mathrm{~g}$ & $168.4 \mathrm{E}$ & $166.6 \mathrm{fg}$ & $173.3 \mathrm{f}$ & $169.6 \mathrm{E}$ \\
\hline Granul $125 \%$ & $218.2 \mathrm{~d}$ & $207.4 \mathrm{e}$ & 213.3 D & $208.3 \mathrm{e}$ & $220.5 \mathrm{~d}$ & 214.5 D \\
\hline Fine $125 \%$ & $237.1 \mathrm{c}$ & $236.3 \mathrm{c}$ & $237.2 \mathrm{C}$ & $237.2 \mathrm{c}$ & $239.4 \mathrm{c}$ & $238.3 \mathrm{C}$ \\
\hline Granul 150\% & $267.3 \mathrm{~b}$ & $243.2 \mathrm{c}$ & 246.3 B & $245.4 \mathrm{c}$ & $269.3 \mathrm{~b}$ & $257.4 \mathrm{~B}$ \\
\hline Fine $150 \%$ & $287.3 \mathrm{a}$ & $261.1 \mathrm{~b}$ & $274.2 \mathrm{~A}$ & $262.8 \mathrm{~b}$ & $289.3 \mathrm{a}$ & $276.5 \mathrm{~A}$ \\
\hline Control & $173.4 \mathrm{f}$ & $153.3 \mathrm{~g}$ & $163.4 \mathrm{EF}$ & $153.5 \mathrm{~h}$ & $174.4 \mathrm{f}$ & 164.4 EF \\
\hline Means & $216.2 \mathrm{~A}^{\prime}$ & $203.5 B^{\prime}$ & & $217.5 \mathrm{~A}^{\prime}$ & 304.3 B' & \\
\hline \multicolumn{7}{|c|}{ Root dry weight (g) } \\
\hline Granul 100\% & $45.8 \mathrm{gh}$ & $46.5 \mathrm{gh}$ & $46.1 \mathrm{~F}$ & $46.0 \mathrm{~g}$ & $46.8 \mathrm{~g}$ & $46.4 \mathrm{~F}$ \\
\hline Fine $100 \%$ & $50.7 \mathrm{fg}$ & $48.3 \mathrm{~g}$ & $49.5 \mathrm{E}$ & $50.9 \mathrm{f}$ & $48.6 \mathrm{fg}$ & $49.8 \mathrm{E}$ \\
\hline Granul 125\% & $64.2 \mathrm{~d}$ & $60.8 \mathrm{e}$ & 62.5 D & $64.7 \mathrm{~d}$ & $61.1 \mathrm{e}$ & 62.9 D \\
\hline Fine $125 \%$ & $69.8 \mathrm{c}$ & $69.4 \mathrm{~cd}$ & 69.6 C & $70.3 \mathrm{c}$ & $69.7 \mathrm{c}$ & $69.9 \mathrm{C}$ \\
\hline Granul $150 \%$ & $78.4 \mathrm{~b}$ & $71.3 \mathrm{c}$ & $74.8 \mathrm{~B}$ & $79.0 \mathrm{~b}$ & $71.9 \mathrm{bc}$ & $75.4 \mathrm{~B}$ \\
\hline Fine $150 \%$ & $84.4 \mathrm{a}$ & $76.6 \mathrm{~b}$ & $80.5 \mathrm{~A}$ & $85.0 \mathrm{a}$ & $77.2 \mathrm{~b}$ & 81.1 A \\
\hline Control & $51.0 \mathrm{f}$ & $44.9 \mathrm{~h}$ & 47.9 EF & $51.2 \mathrm{f}$ & $45.1 \mathrm{~g}$ & 48.2 EF \\
\hline Means & $63.4 \mathrm{~A}^{\prime}$ & 59.6 B' & & $63.8 \mathrm{~A}^{\prime}$ & $60.1 \mathrm{~B}^{\prime}$ & \\
\hline
\end{tabular}

\section{Yield and yield components:}

The data in Table (4) showed that there were significant differences among the tested treatments during the both studied seasons. Paulista cultivar had significant higher total pods yield per plant than Samantha cultivar during the two studied seasons. Regarding the comparison of different phosphorus levels from rock phosphate with the common recommendation by calcium superphosphate (control) the highest phosphorus (150\%) level gave the highest snap bean pods weight per plant followed by $125 \%$ of recommended $\mathrm{P}$, the lowest total pods weight per plant was obtained from control. 
Table 4. Effect of application of different rates of rock phosphate forms as well as two green bean cultivars on total yield per plant, pod length, pod diameter, pod weight and fibers percentages.

\begin{tabular}{|c|c|c|c|c|c|c|}
\hline & \multicolumn{2}{|c|}{ First season $2015 / 2016$} & \multicolumn{3}{|c|}{ Second season $2016 / 2017$} & \multirow[b]{2}{*}{ Means } \\
\hline Treatments & Paulista & Samantha & Means & Paulista & Samantha & \\
\hline \multicolumn{7}{|c|}{ Total yield /plant (g) } \\
\hline Granul 100\% & $373.4 \mathrm{~d}$ & $342.5 \mathrm{e}$ & 357.9 D & $380.9 \mathrm{~d}$ & $354.4 \mathrm{de}$ & $367.6 \mathrm{D}$ \\
\hline Fine $100 \%$ & $412.7 \mathrm{c}$ & $337.1 \mathrm{e}$ & $374.9 \mathrm{CD}$ & $421.0 \mathrm{c}$ & $349.0 \mathrm{e}$ & $384.8 \mathrm{CD}$ \\
\hline Granul 125\% & $412.5 \mathrm{c}$ & $349.6 \mathrm{de}$ & $381.3 \mathrm{C}$ & $423.5 \mathrm{c}$ & $361.6 \mathrm{de}$ & $392.5 \mathrm{C}$ \\
\hline Fine $125 \%$ & $439.0 \mathrm{bc}$ & $350.2 \mathrm{de}$ & $394.3 \mathrm{BC}$ & $451.2 \mathrm{bc}$ & $360.0 \mathrm{de}$ & $405.6 \mathrm{BC}$ \\
\hline Granul $150 \%$ & $457.0 \mathrm{~b}$ & $350.5 \mathrm{de}$ & 403.7 B & $471.2 \mathrm{~b}$ & $362.0 \mathrm{de}$ & 416.6 B \\
\hline Fine $150 \%$ & $513.3 \mathrm{a}$ & $466.4 \mathrm{~b}$ & $489.8 \mathrm{~A}$ & $540.6 \mathrm{a}$ & $479.1 \mathrm{~b}$ & $509.8 \mathrm{~A}$ \\
\hline Control & $292.1 \mathrm{f}$ & $266.3 \mathrm{f}$ & $279.2 \mathrm{E}$ & $301.0 \mathrm{f}$ & $273.3 \mathrm{f}$ & $287.2 \mathrm{E}$ \\
\hline Means & $414.3 \mathrm{~A}^{\prime}$ & 351.8 B' & & $427.1 \mathrm{~A}^{\prime}$ & $362.8 \mathrm{~B}^{\prime}$ & \\
\hline \multicolumn{7}{|c|}{ pod length $(\mathrm{cm})$} \\
\hline Granul 100\% & $15.15 \mathrm{c}$ & $15.26 \mathrm{c}$ & $15.21 \mathrm{C}$ & $15.40 \mathrm{bc}$ & $14.67 \mathrm{~cd}$ & 15.10 DC \\
\hline Fine $100 \%$ & $15.25 \mathrm{c}$ & $15.23 \mathrm{c}$ & $15.24 \mathrm{C}$ & $15.10 \mathrm{bc}$ & $15.03 \mathrm{c}$ & 15.11 DC \\
\hline Granul 125\% & $15.26 \mathrm{c}$ & $15.32 \mathrm{c}$ & 12.29 BC & $15.27 \mathrm{bc}$ & $14.43 \mathrm{~d}$ & 14.85 D \\
\hline Fine $125 \%$ & $15.43 \mathrm{bc}$ & $15.17 \mathrm{c}$ & $15.30 \mathrm{BC}$ & $15.60 \mathrm{ab}$ & $15.07 \mathrm{bc}$ & $15.33 \mathrm{BC}$ \\
\hline Granul 150\% & $15.63 \mathrm{ab}$ & $15.27 \mathrm{c}$ & $15.45 \mathrm{~B}$ & $15.47 \mathrm{~b}$ & $15.63 \mathrm{ab}$ & $15.55 \mathrm{AB}$ \\
\hline Fine $150 \%$ & $15.84 \mathrm{a}$ & $15.42 \mathrm{bc}$ & $15.63 \mathrm{~A}$ & $15.93 \mathrm{a}$ & $15.67 \mathrm{ab}$ & $15.80 \mathrm{~A}$ \\
\hline Control & $15.10 \mathrm{c}$ & $14.51 \mathrm{~d}$ & 14.81 D & $14.53 \mathrm{~d}$ & $15.40 \mathrm{bc}$ & 14.97 D \\
\hline Means & $15.38 \mathrm{~A}^{\prime}$ & 15.17 B' & & $15.33 \mathrm{~A}^{\prime}$ & $15.13 \mathrm{~B}^{\prime}$ & \\
\hline \multicolumn{7}{|c|}{ pod diameter $(\mathrm{cm})$} \\
\hline Granul 100\% & $0.70 \mathrm{de}$ & $0.67 \mathrm{de}$ & $0.68 \mathrm{C}$ & $0.74 \mathrm{~cd}$ & $0.70 \mathrm{~d}$ & $0.72 \mathrm{E}$ \\
\hline Fine $100 \%$ & $0.76 \mathrm{bc}$ & $0.66 \mathrm{e}$ & $0.71 \mathrm{C}$ & $0.77 \mathrm{bc}$ & $0.69 \mathrm{~d}$ & $0.73 \mathrm{DE}$ \\
\hline Granul 125\% & $0.75 \mathrm{bcd}$ & $0.69 \mathrm{de}$ & $0.72 \mathrm{C}$ & $0.79 \mathrm{bc}$ & $0.72 \mathrm{~cd}$ & $0.75 \mathrm{DE}$ \\
\hline Fine $125 \%$ & $0.74 \mathrm{~cd}$ & $0.68 \mathrm{de}$ & $0.72 \mathrm{C}$ & $0.80 \mathrm{~b}$ & $0.75 \mathrm{c}$ & $0.77 \mathrm{BC}$ \\
\hline Granul 150\% & $0.79 \mathrm{ab}$ & $0.71 \mathrm{~d}$ & 0.75 B & $0.84 \mathrm{a}$ & $0.76 \mathrm{bc}$ & 0.79 B \\
\hline Fine $150 \%$ & $0.84 \mathrm{a}$ & $0.75 \mathrm{~cd}$ & $0.79 \mathrm{~A}$ & $0.88 \mathrm{a}$ & $0.79 \mathrm{bc}$ & 0.83 A \\
\hline Control & $0.74 \mathrm{~cd}$ & $0.67 \mathrm{~cd}$ & $0.70 \mathrm{C}$ & $0.77 \mathrm{bc}$ & $0.70 \mathrm{~d}$ & $0.73 \mathrm{DE}$ \\
\hline Means & $0.76 A^{\prime}$ & 0.69 B' & & $0.79 \mathrm{~A}^{\prime}$ & $0.73 \mathrm{~B}^{\prime}$ & \\
\hline \multicolumn{7}{|c|}{ pod weight (gm) } \\
\hline Granul 100\% & $8.50 \mathrm{c}$ & $8.66 \mathrm{c}$ & $8.47 \mathrm{C}$ & $8.90 \mathrm{~d}$ & $8.93 \mathrm{~d}$ & $8.91 \mathrm{D}$ \\
\hline Fine $100 \%$ & $8.45 \mathrm{c}$ & $8.44 \mathrm{c}$ & $8.55 \mathrm{C}$ & $8.87 \mathrm{~d}$ & $9.13 \mathrm{~d}$ & $8.99 \mathrm{D}$ \\
\hline Granul 125\% & $8.78 \mathrm{bc}$ & $8.66 \mathrm{c}$ & $8.75 \mathrm{BC}$ & $9.45 \mathrm{~cd}$ & $9.49 \mathrm{~cd}$ & $9.47 \mathrm{C}$ \\
\hline Fine $125 \%$ & $9.14 \mathrm{bc}$ & $8.72 \mathrm{bc}$ & $9.05 \mathrm{~B}$ & $9.40 \mathrm{~cd}$ & $9.40 \mathrm{~cd}$ & $9.39 \mathrm{C}$ \\
\hline Granul 150\% & $9.26 \mathrm{bc}$ & $8.95 \mathrm{bc}$ & $9.07 \mathrm{~B}$ & $10.29 \mathrm{~b}$ & $9.67 \mathrm{c}$ & $9.97 \mathrm{~B}$ \\
\hline Fine $150 \%$ & $10.67 \mathrm{a}$ & $8.89 \mathrm{bc}$ & $9.99 \mathrm{~A}$ & $11.20 \mathrm{a}$ & $10.13 \mathrm{~b}$ & $10.66 \mathrm{~A}$ \\
\hline Control & $8.50 \mathrm{c}$ & $9.33 \mathrm{c}$ & $8.63 \mathrm{BC}$ & $9.36 \mathrm{~cd}$ & $9.03 \mathrm{~d}$ & $9.19 \mathrm{CD}$ \\
\hline Means & $9.07 \mathrm{~A}^{\prime}$ & $8.79 \mathrm{~B}^{\prime}$ & & $9.63 \mathrm{~A}^{\prime}$ & $9.38 \mathrm{~B}^{\prime}$ & \\
\hline \multicolumn{7}{|c|}{ Fiber content $(\%)$} \\
\hline Granul 100\% & $9.27 \mathrm{a}$ & $9.30 \mathrm{a}$ & $9.28 \mathrm{~A}$ & $9.74 \mathrm{a}$ & $9.77 \mathrm{a}$ & $9.75 \mathrm{~A}$ \\
\hline Fine $100 \%$ & $9.02 \mathrm{~b}$ & $9.22 \mathrm{ab}$ & $9.12 \mathrm{BC}$ & $9.47 \mathrm{~b}$ & $9.68 \mathrm{ab}$ & $9.57 \mathrm{BC}$ \\
\hline Granul 125\% & $8.93 \mathrm{~b}$ & $9.15 \mathrm{ab}$ & 9.04 BCD & $9.38 \mathrm{~b}$ & $9.57 \mathrm{ab}$ & $9.47 \mathrm{BCD}$ \\
\hline Fine $125 \%$ & $8.99 \mathrm{~b}$ & $8.98 \mathrm{~b}$ & $8.98 \mathrm{CD}$ & $9.44 \mathrm{~b}$ & $9.48 \mathrm{~b}$ & 9.46 CD \\
\hline Granul 150\% & $8.82 \mathrm{~b}$ & $9.16 \mathrm{ab}$ & $8.99 \mathrm{CD}$ & $9.26 \mathrm{~b}$ & $9.62 \mathrm{ab}$ & $9.44 \mathrm{CD}$ \\
\hline Fine $150 \%$ & $8.92 \mathrm{~b}$ & $8.92 \mathrm{~b}$ & 8.92 D & $9.37 \mathrm{~b}$ & $9.37 \mathrm{~b}$ & $9.36 \mathrm{D}$ \\
\hline Control & $9.25 \mathrm{ab}$ & $9.17 \mathrm{ab}$ & $9.21 \mathrm{AB}$ & $9.71 \mathrm{ab}$ & $9.63 \mathrm{ab}$ & $9.67 \mathrm{AB}$ \\
\hline Means & $9.03 \mathrm{~B}^{\prime}$ & $9.12 \mathrm{~A}^{\prime}$ & & $9.48 \mathrm{~B}^{\prime}$ & $9.58 \mathrm{~A}^{\prime}$ & \\
\hline
\end{tabular}

The current results show that plants grown with recommended $\mathrm{P}$ fertilizer from calcium superphosphate had the lowest pod yield. Average pod diameter, pod length and pod weight took the same trend like total pod weight. Fiber content took another trend, control treatment had the highest fiber content followed by $100 \%$ of P; the lowest fiber content was obtained by $150 \%$ P. the same results was obtained by (Hossain and Hamid, 2007) who concluded that reduction in pod number was mainly caused by failure 
of fertilization due to production of non-viable pollen grains under such conditions. Similarly, the number of pods per plant was significantly reduced by low $\mathrm{P}$ probably due to increased flower abortion. Generally, yield was mostly reduced at lower $\mathrm{P}$ levels and increased at higher P levels in all tested cultivars. Similarly, (Haugen and Bennink, 2003) observed that reduction in yield is largely due to reduction in number of pods per plant. It could be concluded that the phosphorus fertilizer had a major effect on the productivity of bean plant, hence increased total yield and its components. It may be attributed to the enhancement of phosphorus on the plant growth and it's reflected on the yield. Many investigators had obtained a similar trend of results (Farag et al., 2010 and Marschner, 1995). (Roy and Parthasarathy, 1999) who found that green bean pod yield was the highest with $120 \mathrm{~kg}$ units per hectare of $\mathrm{P}$ compared to lower rates. Generally, the amount of photosynthate available for biomass production is related to the plant leaf area and the photosynthetic rate of the crop. The quality of green bean expressed as fiber contents was enhanced with increasing concentration of foliar application with nutrients. This may be due to increasing plant availability of nutrients led to reduce fiber contents (Tantawy et al., 2009). This increment in dry matter accumulation with application of $\mathrm{P}$ fertilizer might be due to the adequate supply of phosphorus which attributed to an increase plant canopy. This in turn increased photosynthetic area and number of pods per plant. Furthermore, (Moniruzzaman et. al., 2008) who reported that average weight and length in green bean was significantly increased as phosphorus application rate increased from 0 to $80 \mathrm{~kg} \mathrm{P}_{2} \mathrm{O}_{5} \mathrm{ha}^{-1}$, and remained relatively constant at higher $\mathrm{P}$ rates $\left(120 \mathrm{~kg} \mathrm{P}_{2} \mathrm{O}_{5}\right.$ ha $^{-}$ ${ }^{1}$ ). The significant variation between the two cultivars might be attributed to the genetic potential of the cultivars and the cultivars and environment responses (khan et al., 2003).

\section{Nutrient percentage:}

The obtained results in Table 5 showed that the phosphorus and bean cultivar treatments significantly affected the uptake of NPK by bean plant during the two growing seasons. Paulista cultivar had higher NPK percentage than Samantha during the two studied seasons. Increasing uptake of P level led to increase nitrogen percentage in bean leaves. Applied phosphorus by using fine or granule natural rock phosphate was significantly affected NPK percentage in bean leaves than using calcium super phosphate as source of phosphorus fertilizer (Talbi-Zribi et al., 2014 and Cakmak et. al., 1994).

Table 5. Effect of application of different rates of rock phosphate forms as well as two green bean cultivars on nitrogen, phosphorus, potassium percentages of leaves.

\begin{tabular}{|c|c|c|c|c|c|c|}
\hline & \multicolumn{2}{|c|}{ First season 2015/2016 } & \multicolumn{3}{|c|}{ Second season $2016 / 2017$} & \multirow[b]{2}{*}{ Means } \\
\hline Treatments & Paulista & Samantha a & Means & Paulista & Samantha & \\
\hline \multicolumn{7}{|c|}{$\mathbf{N} \%$} \\
\hline Granul 100\% & $2.67 \mathrm{de}$ & $2.63 \mathrm{e}$ & $2.65 \mathrm{C}$ & $2.80 \mathrm{e}$ & $2.75 \mathrm{f}$ & $2.77 \mathrm{~F}$ \\
\hline Fine $100 \%$ & $2.67 \mathrm{de}$ & $2.68 \mathrm{de}$ & $2.68 \mathrm{C}$ & $2.81 \mathrm{e}$ & $2.81 \mathrm{e}$ & $2.80 \mathrm{E}$ \\
\hline Granul $125 \%$ & $2.69 \mathrm{de}$ & $2.70 \mathrm{~d}$ & $2.69 \mathrm{C}$ & $2.86 \mathrm{~d}$ & $2.85 \mathrm{de}$ & $2.85 \mathrm{D}$ \\
\hline Fine $125 \%$ & $2.79 \mathrm{c}$ & $2.73 \mathrm{~cd}$ & $2.76 \mathrm{~B}$ & $2.93 \mathrm{c}$ & $2.87 \mathrm{~d}$ & $2.89 \mathrm{C}$ \\
\hline Granul 150\% & $2.84 \mathrm{bc}$ & $2.77 \mathrm{~cd}$ & $2.80 \mathrm{~B}$ & $2.98 \mathrm{~b}$ & $2.91 \mathrm{~cd}$ & 2.94 B \\
\hline Fine $150 \%$ & $3.17 \mathrm{a}$ & $2.87 \mathrm{~b}$ & $3.02 \mathrm{~A}$ & $3.39 \mathrm{a}$ & $3.02 \mathrm{~b}$ & $3.20 \mathrm{~A}$ \\
\hline Control & $2.61 \mathrm{e}$ & $2.68 \mathrm{de}$ & $2.64 \mathrm{C}$ & $2.69 \mathrm{~g}$ & $2.74 \mathrm{f}$ & $2.71 \mathrm{G}$ \\
\hline Means & $2.78 \mathrm{~A}^{\prime}$ & $2.72 \mathrm{~B}^{\prime}$ & & $2.92 \mathrm{~A}^{\prime}$ & $2.85 \mathrm{~B}^{\prime}$ & \\
\hline \multicolumn{7}{|c|}{$\mathbf{P} \%$} \\
\hline Granul 100\% & $0.42 \mathrm{e}$ & $0.38 \mathrm{~d}$ & $0.40 \mathrm{D}$ & $0.44 \mathrm{e}$ & $0.41 \mathrm{f}$ & $0.42 \mathrm{D}$ \\
\hline Fine $100 \%$ & $0.42 \mathrm{e}$ & $0.38 \mathrm{~d}$ & 0.39 D & $0.45 \mathrm{de}$ & $0.40 \mathrm{fg}$ & $0.42 \mathrm{D}$ \\
\hline Granul 125\% & $0.47 \mathrm{c}$ & $0.43 \mathrm{de}$ & $0.45 \mathrm{C}$ & $0.49 \mathrm{c}$ & $0.45 \mathrm{de}$ & $0.47 \mathrm{C}$ \\
\hline Fine $125 \%$ & $0.46 \mathrm{~cd}$ & $0.45 \mathrm{~d}$ & $0.46 \mathrm{C}$ & $0.50 \mathrm{c}$ & $0.47 \mathrm{~d}$ & $0.48 \mathrm{C}$ \\
\hline Granul $150 \%$ & $0.51 \mathrm{~b}$ & $0.47 \mathrm{c}$ & 0.49 B & $0.54 \mathrm{~b}$ & $0.49 \mathrm{c}$ & $0.51 \mathrm{~B}$ \\
\hline Fine $150 \%$ & $0.55 \mathrm{a}$ & $0.48 \mathrm{c}$ & $0.51 \mathrm{~A}$ & $0.58 \mathrm{a}$ & $0.50 \mathrm{c}$ & $0.53 \mathrm{~A}$ \\
\hline Control & $0.38 \mathrm{~d}$ & $0.37 \mathrm{~d}$ & $0.37 \mathrm{E}$ & $0.40 \mathrm{fg}$ & $0.39 \mathrm{~g}$ & $0.39 \mathrm{E}$ \\
\hline Means & $0.46 \mathrm{~A}^{\prime}$ & $0.42 \mathrm{~B}^{\prime}$ & & $0.48 \mathrm{~A}^{\prime}$ & $0.44 \mathrm{~B}^{\prime}$ & \\
\hline \multicolumn{7}{|c|}{ K \% } \\
\hline Granul 100\% & $2.54 \mathrm{ef}$ & $2.55 \mathrm{e}$ & $2.55 \mathrm{~F}$ & $2.66 \mathrm{~g}$ & $2.67 \mathrm{~g}$ & $2.66 \mathrm{G}$ \\
\hline Fine $100 \%$ & $2.68 \mathrm{~d}$ & $2.51 \mathrm{f}$ & $2.59 \mathrm{E}$ & $2.80 \mathrm{de}$ & $2.65 \mathrm{~g}$ & $2.72 \mathrm{~F}$ \\
\hline Granul 125\% & $2.71 \mathrm{~cd}$ & $2.58 \mathrm{e}$ & $2.65 \mathrm{D}$ & $2.85 \mathrm{~cd}$ & $2.73 \mathrm{f}$ & 2.79 D \\
\hline Fine $125 \%$ & $2.74 \mathrm{c}$ & $2.70 \mathrm{~cd}$ & $2.72 \mathrm{C}$ & $2.88 \mathrm{c}$ & $2.83 \mathrm{~d}$ & $2.85 \mathrm{C}$ \\
\hline Granul $150 \%$ & $2.85 \mathrm{~b}$ & $2.68 \mathrm{~d}$ & $2.77 \mathrm{~B}$ & $2.99 \mathrm{~b}$ & $2.82 \mathrm{~d}$ & $2.90 \mathrm{~B}$ \\
\hline Fine $150 \%$ & $2.91 \mathrm{a}$ & $2.74 \mathrm{c}$ & $2.83 \mathrm{~A}$ & $3.05 \mathrm{a}$ & $2.88 \mathrm{c}$ & $2.96 \mathrm{~A}$ \\
\hline Control & $2.64 \mathrm{~d}$ & $2.64 \mathrm{~d}$ & 2.94 D & $2.74 \mathrm{ef}$ & $2.77 \mathrm{e}$ & $2.75 \mathrm{D}$ \\
\hline Means & $2.73 \mathrm{~A}^{\prime}$ & $2.63 \mathrm{~B}^{\prime}$ & & $2.85 \mathrm{~A}^{\prime}$ & $2.76 \mathrm{~B}$ & \\
\hline
\end{tabular}


Increasing percentage of rock phosphate led to increase NPK percentage up to $150 \%$. Increasing phosphorus percentage led to increase both nitrogen and potassium percentage in bean leaves (Hinsinger, 2001, Kouas et al., 2008 and Farag et al., 2010).

The Economic consideration of different applied phosphorus treatments

The average costs of using different phosphorus levels and sources for two green beans cultivars during the two seasons are shown in Table (6). The economic evaluation considered the cost of granule 600 L.E and fine rock phosphate 550 as well as cost of calcium superphosphate is 1100 L.E/ton (average for the two seasons). In this study all the other costs of production such as labor, inputs, irrigation etc. are not included because they were the same as in different tested treatments.
The application phosphorus cost per greenhouse was calculated under different applications (100-125 and $150 \%$ of recommended dose). The highest phosphorus costs was under control treatment (100 L.E/ GH) followed by $150 \%$ (60 LE/GH) of granule rock phosphate treatment; the lowest cost was calculated for $100 \%$ fine rock phosphate (37 L.E/GH). The highest net income was gained by $150 \%$ of fine rock phosphate with Paulista cultivar followed by using $150 \%$ of fine rock phosphate with Samantha cultivar. The lowest net income was obtained by control treatment with the both tested cultivars. The yield increased with the increasing in phosphorus levels from 100 to $150 \mathrm{~kg} \mathrm{P}$ per GH, these increasing of the yield with the $150 \mathrm{~kg}$ P per GH was covered the additional cost of fertilizer with plenty incremental income. Fine rock phosphate gave higher yield than granule phosphate while the cost of fine rock phosphate is lower than granule one.

Table 6. Average economic analysis of green bean production under differences on gross profit due to the application of different phosphorus levels during the two seasons.

\begin{tabular}{|c|c|c|c|c|c|c|c|c|c|c|c|}
\hline$\underbrace{\grave{\Xi}}$ & $\begin{array}{c}\mathbf{P} \\
\text { Source }\end{array}$ & P \% & $\begin{array}{c}\text { Avge. } \\
\text { yield } \\
\text { kg/pot }\end{array}$ & $\begin{array}{l}\text { Yield } \\
\text { Kg/ } \\
\text { GH }\end{array}$ & $\begin{array}{c}\text { Average } \\
\text { price } \\
\text { LE/ kg }\end{array}$ & $\begin{array}{c}\text { Grass } \\
\text { income } \\
\text { GH }\end{array}$ & $\begin{array}{c}\text { P Unit } \\
\text { Cost } \\
\text { LE/ Ton }\end{array}$ & $\begin{array}{l}\text { Applied } \\
\text { P } \\
\text { kg/ GH }\end{array}$ & $\begin{array}{l}\text { P total } \\
\text { Cost } \\
\text { L. E/ } \\
\text { GH }\end{array}$ & $\begin{array}{c}\text { Net } \\
\text { income } \\
\text { L. E/ } \\
\text { GH }\end{array}$ & $\begin{array}{c}\text { Incremental } \\
\text { income } \\
\text { L. E / GH }\end{array}$ \\
\hline \multirow{6}{*}{ 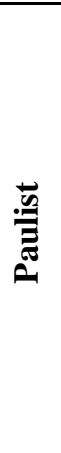 } & \multirow{3}{*}{ 光 } & $100 \%$ & 0.38 & 2514 & \multirow{14}{*}{5.00} & 12572 & 600 & 67 & 40 & 12532 & 2746.4 \\
\hline & & $125 \%$ & 0.42 & 2787 & & 13934 & 600 & 83 & 50 & 13884 & 4098.1 \\
\hline & & $150 \%$ & 0.46 & 3094 & & 15471 & 600 & 100 & 60 & 15411 & 5624.9 \\
\hline & \multirow{3}{*}{ 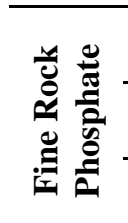 } & $100 \%$ & 0.42 & 2779 & & 13896 & 550 & 67 & 37 & 13859 & 4073.1 \\
\hline & & $125 \%$ & 0.45 & 2967 & & 14837 & 550 & 83 & 46 & 14792 & 5005.7 \\
\hline & & $150 \%$ & 0.53 & 3513 & & 17566 & 550 & 100 & 55 & 17511 & 7725.0 \\
\hline \multirow{8}{*}{ 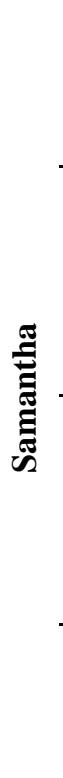 } & è & $100 \%$ & 0.30 & 1977 & & 9885 & 1100 & 91 & 100 & 9786 & 0.0 \\
\hline & \multirow{3}{*}{ 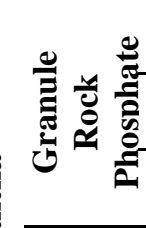 } & $100 \%$ & 0.35 & 2323 & & 11616 & 600 & 67 & 40 & 11576 & 2681.4 \\
\hline & & $125 \%$ & 0.36 & 2371 & & 11854 & 600 & 83 & 50 & 11804 & 2909.7 \\
\hline & & $150 \%$ & 0.36 & 2375 & & 11876 & 600 & 100 & 60 & 11816 & 2921.4 \\
\hline & \multirow{3}{*}{ 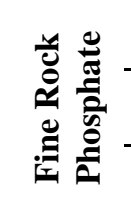 } & $100 \%$ & 0.34 & 2287 & & 11436 & 550 & 67 & 37 & 11399 & 2504.7 \\
\hline & & $125 \%$ & 0.36 & 2367 & & 11837 & 550 & 83 & 46 & 11791 & 2897.2 \\
\hline & & $150 \%$ & 0.47 & 3152 & & 15759 & 550 & 100 & 55 & 15704 & 6809.9 \\
\hline & ن̈ & $100 \%$ & 0.27 & 1799 & & 8994 & 1100 & 91 & 100 & 8894 & 0.0 \\
\hline
\end{tabular}




\section{Conclusion}

Results from this study showed that the application of rock phosphate as a source of $\mathrm{P}$, improves the growth and seed yield of green beans. However, using $150 \%$ of fine rock phosphate with Paulista cultivars gives the best growth and yields in comparison with using calcium superphosphate with the recommended dose.

\section{References}

Adediran, J A. and Sobulo, R.A. (1997). The potentials and use of rock phosphates in the Sub Saharan Africa. A case study in Nigeria. In: (Agboola et al.,) Proceedings of 3 rd All African Soil Society, University of Ibadan, Ibadan. Nigeria, August1995. Pp 295 - 305.

Adetunji, M.T. 2005. Soil quality for ecological security and sustainable agriculture. UNAAB Inaugural Lecture, Series No 19, University of Agriculture, Abeokuta, 39p.

Akande, M. O.; F. I. Oluwatoyinbo, C. O. Kayode and F. A. Olowookere,(2008). Effects of Ogun phosphate rock amended with different levels of cowdung on the growth and yield of maize okra intercrop relayed with cowpea. African Journal of Biotechnology 7 (17): 3039-3043.

Allen, S. E. (1974). Chemical Analysis of Ecological Materials. Black-Well, Oxford, p: 565.

A.O.A.C. (1990). Official Methods of Analysis of the Association of Official Analytical Chemists. $15^{\text {th }}$ ed. Published by the Association of Official Analytical Chemists, Anlington, Virginia 2201, USA.

Bargaz, A., R.M.A.Nassar, M.M. Rady, M.S. Gaballah, S.M.Thompson, , M. Brestic and MT., Abdelhamid, (2016). Improved salinity tolerance by phosphorus fertilizer in two Phaseolus vulgaris recombinant inbred lines contrasting in their $\mathrm{P}$ efficiency. - Journal of Agronomy and Crop Science 202(6): 497-507.

Bhuiyan M.M.H., M.M. Rahman,F. Afroze, G.N.C. Sutradhar and M.S.I. Bhuiyan, (2008). Effect of Phosphorus, Molybdenum and Rhizobium Inoculation on Growth and Nodulation of Mungbean. J Soil Nature 2(2): 25-30

Cakmak, I., C. Hengeler and H. Marschner (1994). Partitioning of shoot and root dry matter and carbohydrates in bean plants suffering from phosphorus, potassium and magnesium deficiency. - Journal of Experimental Botany 45(9): 1245-1250.

Chapman, H. D. and P. F. Pratt (1961). Methods of analysis for soil, plant, and water. University of California, Division of Agric Sci.

CIMMYT (1988) An Economic Training Manual: From Agronomic Data to Farmer ecommendations, Mexico, 1-25.
FAO, (1980). Soil and Plant Analysis. Soils Bulletin $38 / 2,250$.

FAO Stat. (2013): Agriculture Organization of the United Nations, 2013. FAO, Retrieved from http://faostat3. fao.org/faostatgateway/go/to/download/Q/QC/S. Acceso, 20.

Farag, A. A., M. A. A. Abdrabbo and M. K Hassanein. (2010). Response of Cucumber for Mulch Colors and Phosphorus Levels under Greenhouse. Egypt J. Hort. 37, 1:53-64.

Food and Agriculture Organization (FAO). (1980). Soil and Plant Analysis. Soils Bulletin, 38: 242250.

Haugen, L. A. and M. R. Bennink,, (2003). Composition of black beans and navy beans (Phaseolus vulgaris) reduced azoxymethane induced colon cancer in rats. Nutrition Cancer 44: $60-65$.

Hinsinger, P., 2001. Bioavailability of soil inorganic $\mathrm{P}$ in the rhizosphere as affected by root-induced chemical changes: a reviw. Plant and soil, 237(2) 173-195 Dec 2001.

Hossain, M. A. and A.Hamid, (2007). Influence of N and $\mathrm{P}$ fertilizer application on root growth, leaf photosynthesis and yield performance of groundnut. Bangladesh Journal of Agricultural Research 32(3): 369-374.

Johnston, A.E., and I.Steen, (2000): Understanding phosphorus and its use in agriculture. European Fertilizer Manufacturers' Association. http://www.fertilizerseurope.com/

fileadmin/user_upload/publications/agriculture_p ublications/EFMA_Phosphorus_booklet__2_.pdf (Internet: 3.Feb., 2018).

Kavanova' M., F.ALattanzi., A.A. Grimoldi, and H. Schnyder (2006). Phosphorus Defi ciency Decreases Cell Division and Elongation in Grass Leaves. Plant Physiol 141:766-775

Khan, M. B., M. Asif, N. Hussain, and M. Aziz. 2003. Impact of Different Levels of Phosphorus on Growth and Yield of Mungbean Genotypes. Asian Journal of Plant Sciences 2 (9): 677-679

Kouas S, N. Alkama ,C. Abdelly and J.J. Drevon. (2008). Proton release by nodulated roots varies among common bean genotypes (Phaseolus vulgaris) under phosphorus deficiency. Journal of Soil Science and Plant Nutrition; 171: 242-248

Liu, J. Z., Z. S. Li, and J. Y. Li, (1994). Utilization of plant potentialities to enhance the bio-efficiency of phosphorus in soil. Eco- Agriculture Research 2: 16-23.

Marschner 1995. Mineral nutrition of higher plants. 2nd Edition, Academic Press, San Diego,889 pp.

Ministry of Agriculture and Land Reclamation (2013) Soils, Water and Environment Research Institute, Agricultural Research Centre.

Mongi, H. O. (1974). Lime, nitrogen and phosphorus effects on some chemical characteristics of an oxisol, leaf element content and yield in upland rice and maize at Morogoro. Unpublished Thesis 
for Award of PhD. at University of Dar esSalaam, Tanzania. 17- 47 pp.

Moniruzzaman M., M. R.Islam and J. Hassan (2008). Eff ect of N P K S Zn and B on yield attributes and yield of french bean in south eastern hilly region of Bangladesh. J Agric Rural Dev 6(1\&2): 75-82.

Nikolay Vassilev, Irena Franco, Maria Vassileva and Rosario Azcon, 1996. Improved plant growth with

Raboy V. (2003). Molecules of interest: myo-inositol1, 2, 3, 4, 5, 6-hexakisphosphate. Phytochem 64:1033-1043.

Raghothama, K.G. (1999): Phosphate acquisition. Annual Review of Plant Physiology and Plant Molecular Biology 50: 665-693

Robinson, H. F., R. E. Comstock, and P. H. Harvey, (1981). Genotypic and Phenotypic correlations in corn and their implications in selection. Agronomy Journal 43 : 282-287.

Roy, N.R. and V.A. Parthasarathy, (1999). Note on phosphorus requirement of French bean Phaseolus vulgaris, L. varieties planted at different dates. Indian J. Horticulture, 56(4): 317-320.

Talbi-Zribi, O., H. Houmani, S.Kouas, I. Slama, R. Ksouri, and C. Abdelly, (2014): Comparative study of the interactive effects of salinity and phosphorus availability in wild (Hordeum maritimum) and cultivated carley (H. vulgare). Journal of Plant Growth Regulation (33): 860-870.

Tantawy, A.S., A.M.R. Abdel-Mawgoud, Hoda A.M. Habib and Magda M. Hafez. (2009). Growth, Productivity and Pod Quality Responses of Green Bean Plants Phaseolus vulgaris to Foliar Application of Nutrients and Pollen Extracts. Research Journal of Agriculture and Biological Sciences, 5(6): 1032-1038

Tesfaye M.J., D.L.Liu, C.P. Allan and R. Vance, (2007).Genomic and genetic control of phosphate stress in legumes. Plant Physiol., 144. 594603.2007.

Wang, Q. R., J. Y. Liand Z. S. Li, (1998). Dynamics and prospect on study of high acquisition of soil unavailable phosphorus by plant. Plant Nutrition Fertility Science 4: 107-116

Watanabe, F. S. and S. R.Olsen (1965). Test of an ascorbic acid method for determining phosphorus in water and $\mathrm{Na} \mathrm{HCO}_{3}$ extracts from soil. Soil Sci. Soc. Amer. Proc., 29: 677-678. 


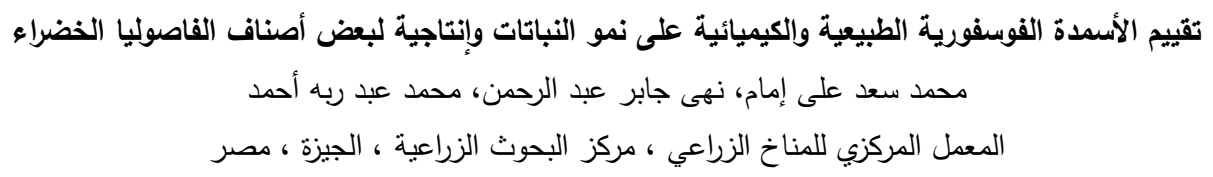

أجريت التجربة الميدانية خلال موسمين متتاليين للموسم 2016/2015 و 2017/2016 في المعمل المركزي للمناخ الزراعي ، مركز البحوث الزراعية ، الدقي ، محافظة الجيزة ، مصر • لدراسة تأثير صنفين من أصناف الفاصوليا الخضراء (بوليستا و سامنتا) مع ثلاثة مستويات من صخر الفوسفات (ناعم وحبييات) كمصدر طبيعي من الفسفور مخلوط فى بيئيه رملية مقارنة مع سوبر فوسفات الكالسيوم بالنسب الموصى بها على الفى

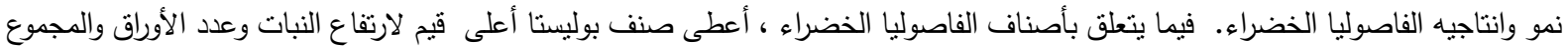
الخضرى والجذرى الطازج والجاف من صنف سامنتا. أنتج صنف بوليستا أعلى القيم من محصول القرون لكل نبات خلال موسمين الزراعة. أدت بـاء زيادة مستوى الفسفور (ناعم وحبيات من صخر الفوسفات) إلى 150٪ الى تحسين محصول القرون مع صنفى الفاصوليا الخضراء تحت الدراسة أعلى نمو خضرى ومحصول تم الحصول عليها من المعامله 150 ٪ من صخر الفوسفات مع صنف بوليستا .أظهر التحليل الكيماوى أن زيادة مستوى الفسفور من مصدر صخر الفسفور أدى إلى زيادة نسبة النيتروجين والفسفور والبوتاسيوم في أوراق الفاصوليا الخضراء مقارنة مع جرعة

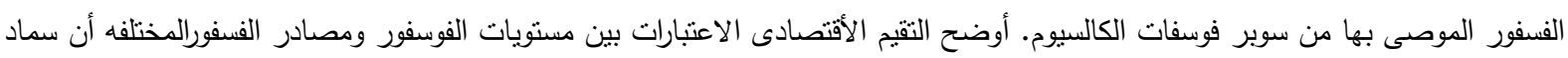
صخر الفوسفات الناعم مع الصنف بوليستا أعطى أعلى عائد صافى لكل صوبه. 